Discrete Comput Geom 35:255-267 (2006)

DOI: $10.1007 / \mathrm{s} 00454-005-1172-4$

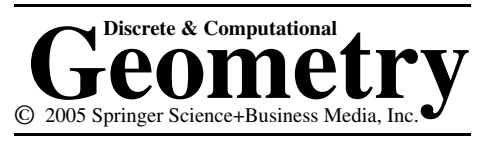

\title{
Compact Packings of the Plane with Two Sizes of Discs*
}

\author{
Tom Kennedy \\ Department of Mathematics, University of Arizona, \\ Tucson, AZ 85721, USA \\ tgk@math.arizona.edu
}

\begin{abstract}
We consider packings of the plane using discs of radius 1 and $r$. A packing is compact if every disc $D$ is tangent to a sequence of discs $D_{1}, D_{2}, \ldots, D_{n}$ such that $D_{i}$ is tangent to $D_{i+1}$. We prove that there are only nine values of $r$ with $r<1$ for which such packings are possible. For each of the nine values we describe the possible compact packings.
\end{abstract}

\section{Introduction}

A packing of the plane with discs is said to be compact if every disc $D$ is tangent to a sequence of discs $D_{1}, D_{2}, \ldots, D_{n}$ such that $D_{i}$ is tangent to $D_{i+1}$ for $i=1,2, \ldots, n$ with $D_{n+1}=D_{1}$ [3]. If we pack the plane using discs of the same radius, then the only possible compact packing is the triangular lattice in which each disc is surrounded by six discs tangent to it. In this paper we ask what compact packings are possible if we pack the plane using discs of radius 1 and $r$ with $r<1$. We do not impose any condition on the relative number of discs of the two radii except to require that discs of both sizes be present. We prove that there are only nine values of $r$ for which a compact packing is possible. For these values we describe the possible compact packings.

The nine values of $r$ which allow compact packings are given in Table 1. Examples of compact packings for each of the nine values are given in Fig. 1. Compact packings for seven of the nine possible values of $r$ (all but $c_{2}$ and $c_{5}$ ) appear in L. Fejes Tóth's classic book [2] and in [7] and [9]. A packing with $r=c_{5}$ appears in [8].

An interesting question is to find (for each $r$ ) the densest packing of the plane using discs of radius 1 and $r$. For six of the above values of $r\left(c_{1}, c_{3}, c_{4}, c_{6}, c_{7}, c_{8}\right)$, Heppes has proved that the densest packing is a compact packing [5], [6]. We expect that for the

* This work was supported by the National Science Foundation (DMS-0201566). 
Table 1. The nine values of $r$ for which a compact packing using discs of radius 1 and $r$ is possible.*

\begin{tabular}{lcccc}
\hline & Decimal & Exact & $i j k$ & Sequence \\
\hline$c_{1}$ & 0.637556 & $r^{4}-10 r^{2}-8 r+9=0$ & 320 & $1111 r$ \\
$c_{2}$ & 0.545151 & $P(r)=0$ & 221 & $111 r r$ \\
$c_{3}$ & 0.533296 & $8 r^{3}+3 r^{2}-2 r-1=0$ & 140 & $1 r 1 r 1$ \\
$c_{4}$ & 0.414214 & $\sqrt{2}-1$ & 400 & 1111 \\
$c_{5}$ & 0.386106 & {$[2 \sqrt{3}+1-2 \sqrt{1+\sqrt{3}}] / 3$} & 122 & $1 r r r 1$ \\
$c_{6}$ & 0.349198 & $\sin (\pi / 12) /(1-\sin (\pi / 12))$ & 041 & $1 r r 1 r$ \\
$c_{7}$ & 0.280776 & $(\sqrt{17}-3) / 4$ & 220 & $111 r$ \\
$c_{8}$ & 0.154701 & $2 \sqrt{3} / 3-1$ & 300 & 111 \\
$c_{9}$ & 0.101021 & $5-2 \sqrt{6}$ & 121 & $11 r r$ \\
\hline
\end{tabular}

*The decimal expressions are of course approximations. For six values of $r$ we give an explicit exact expression. For the other three we give polynomials which have $c_{i}$ as a root. In particular, $c_{2}$ is a root of $P(r)=(7+4 \sqrt{3}) r^{4}+(20+12 \sqrt{3}) r^{3}+$ $(6+4 \sqrt{3}) r^{2}+(-20-4 \sqrt{3}) r+3$. The last two columns give information on the sequence of discs that can appear around a small disc. These two columns are explained in later sections.

other three values of $r$ the densest packing is also a compact packing. Rigorous bounds on the densest packing may be found in [1] and [4] and a non-rigorous study is in [8].

\section{Values of $\boldsymbol{r}$ that Allow Compact Packings}

Theorem 1. The nine values of $r$ given in Table 1 are the only values in $(0,1)$ for which there exists a compact packing of the plane using discs of radius $r$ and 1 .

This section is devoted to the proof of this theorem. The idea is simple. Consider the center of some disc and the angles that are formed by drawing line segments from the center of the disc to the centers of the discs that are tangent to the original disc. There are only a few possible values for these angles and they are functions of $r$. The sum of the angles around the center must be $2 \pi$, and this constrains $r$.

To begin the proof, consider three discs that are tangent to each other and the angles in the triangle whose vertices are their centers. If all three discs have radius 1 or all three have radius $r$, then the angles are all $\pi / 3$. If two of the discs have radius 1 and one has radius $r$, then the triangle has one angle of $\alpha$ and two of $\alpha^{\prime}$ where

$$
\cos \left(\alpha^{\prime}\right)=1 /(1+r), \quad \alpha=\pi-2 \alpha^{\prime} .
$$

If two of the discs have radius $r$ and one has radius 1, then the triangle has one angle of $\beta$ and two of $\beta^{\prime}$ where

$$
\cos \left(\beta^{\prime}\right)=r /(1+r), \quad \beta=\pi-2 \beta^{\prime} .
$$

Thus in a compact packing the angles around a disc of radius $r$ can only be $\pi / 3, \alpha$ and $\beta^{\prime}$, and the angles around a disc of radius 1 can only be $\pi / 3, \alpha^{\prime}$ and $\beta$. Since the angles around a disc must sum to $2 \pi$, there must exist non-negative integers $i, j, k$ such that

$$
i \alpha+j \beta^{\prime}+k \pi / 3=2 \pi
$$




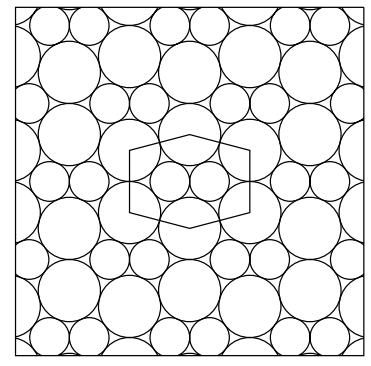

(a) $r=c_{1}=0.637556 \cdots$

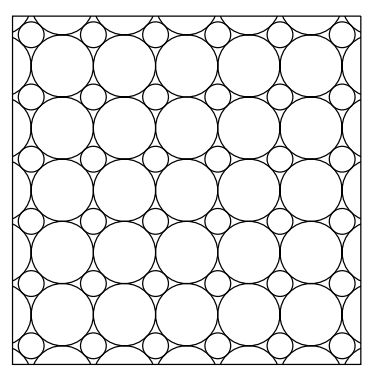

(d) $r=c_{4}=0.414214 \ldots$

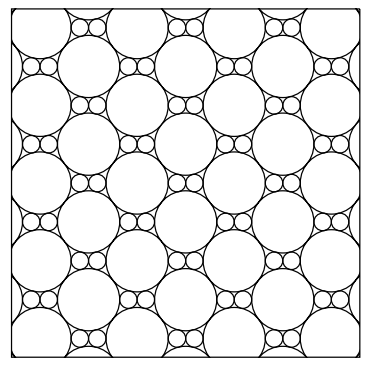

(g) $r=c_{7}=0.280776 \cdots$

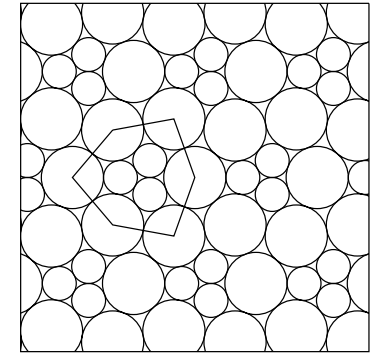

(b) $r=c_{2}=0.545151 \cdots$.

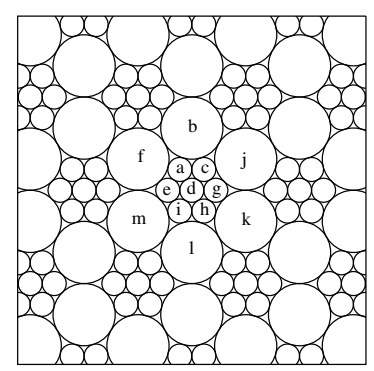

(e) $r=c_{5}=0.386106 \cdots$

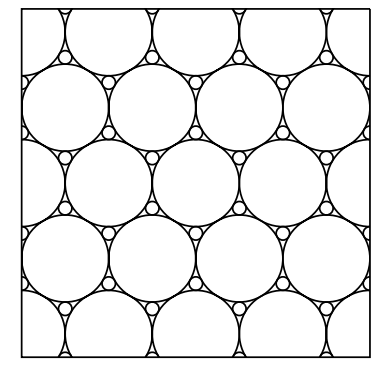

(h) $r=c_{8}=0.154701 \cdots$

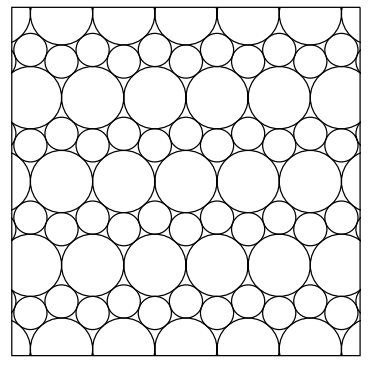

(c) $r=c_{3}=0.533296 \ldots$

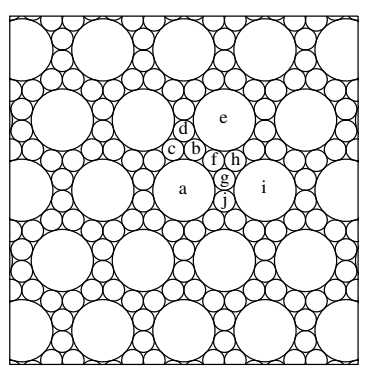

(f) $r=c_{6}=0.349198 \ldots$

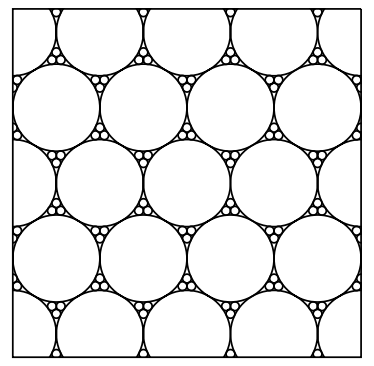

(i) $r=c_{9}=0.101021 \ldots$

Fig. 1. A compact packing is shown for each of the nine values of $r$ which admit compact packings.

and non-negative integers $l, m, n$ such that

$$
l \alpha^{\prime}+m \beta+n \pi / 3=2 \pi .
$$

For every value of $r$ there is a trivial solution of both of these equations, namely $i=$ $j=l=m=0, k=n=6$. In a compact packing that contains discs of both radii, there must be at least one pair of discs of different radii that are tangent, and so there must be at least one solution of (3) other than the trivial one and at least one solution of (4) other than the trivial one. We start by determining when (3) has solutions. 
Define

$$
F_{i j k}(r)=i \alpha+j \beta^{\prime}+k \pi / 3 .
$$

Equations (1) and (2) imply that $\alpha$ and $\beta^{\prime}$ are decreasing functions of $r$, and so $F_{i j k}(r)$ is also decreasing for each choice of $i j k$. This implies that for each choice of $i j k$ there is at most one value of $r$ for which $F_{i j k}(r)=2 \pi$.

As $r$ goes to zero, $\alpha$ converges to $\pi$ and $\beta^{\prime}$ converges to $\pi / 2$. So

$$
\lim _{r \rightarrow 0} F_{i j k}(r)=i \pi+j \pi / 2+k \pi / 3 .
$$

When $r=1$ all the angles are $\pi / 3$, so

$$
F_{i j k}(1)=(i+j+k) \pi / 3 \text {. }
$$

So there is an $r \in(0,1)$ with $F_{i j k}(r)=2 \pi$ if and only if we have $6 i+3 j+2 k>12$ and $i+j+k<6$. Thus there are only a finite number of $i, j, k$ for which (3) might have a solution. There are further constraints on $i, j, k$. Recall that the sides adjacent to the angle $\alpha$ both have length $1+r$, the sides adjacent to $\beta$ have lengths $1+r$ and $2 r$ and the sides adjacent to $\pi / 3$ both have length $2 r$. This implies that $j$ must be even, and if $j$ equals zero, then one of $i$ and $k$ must be zero. It is now trivial to check that the only possibilities for $i j k$ are the nine listed in the penultimate column of the table and one additional case, $i=5, j=0, k=0$. For this last case, $F_{i j k}(r)=2 \pi$ at $r=(1-\sin (\pi / 5)) / \sin (\pi / 5)=0.701303 \cdots$.

The case of $r=(1-\sin (\pi / 5)) / \sin (\pi / 5)$ can be ruled out by considering (4). We compute $\alpha^{\prime}$ and $\beta$ and check if (4) is satisfied for any choice of $l, m, n$. We find that it is not. Thus the only $r$ which might allow compact packings are those shown in the table. The explicit packings shown in Fig. 1(a)-(i) show that compact packings with these nine values of $r$ are indeed possible. It is straightforward to use the figures to compute the nine values of $r$ given in the table. This completes the proof of Theorem 1.

\section{The Compact Packings}

In this section we describe the possible compact packings for $r=c_{1}$ to $c_{9}$. It will be helpful to put (3) and (4) in a slightly different form. Consider the triangle formed by the centers of discs with radii $r_{1}, r_{2}, r_{3}$ which are tangent to each other. Let $\theta\left(r_{1}, r_{2}, r_{3}\right)$ denote the angle at the center of the disc of radius $r_{1}$. Depending on the values of $r_{1}, r_{2}, r_{3}, \theta\left(r_{1}, r_{2}, r_{3}\right)$ is either $\pi / 3, \alpha, \alpha^{\prime}, \beta$ or $\beta^{\prime}$. Now let $D$ be a disc with radius $r_{0}$ and let $D_{1}, D_{2}, \ldots, D_{n}$ be the discs that are tangent to $D$ and such that $D_{i}$ is tangent to $D_{i+1}$. Let $r_{1}, r_{2}, \ldots, r_{n}$ be their radii. Then we must have

$$
\sum_{i=1}^{n} \theta\left(r_{0}, r_{i}, r_{i+1}\right)=2 \pi,
$$

where $r_{n+1}=r_{1}$. Of course each $r_{i}$ is either 1 or $r$. So for $r_{0}$ equal to 1 or $r$, we want to find all finite sequences $r_{1}, r_{2}, \ldots, r_{n}$ consisting of 1's and $r$ 's such that the above equation is satisfied. (As we noted before, we seek solutions beyond the trivial ones with $n=6$ and $r_{1}=\cdots=r_{6}=r_{0}$ which are solutions for all $r$.) 
We write the cases for which (8) is satisfied in the form

$$
r_{0}: r_{1} r_{2} r_{3} \cdots r_{n}
$$

meaning that a disc of radius $r_{0}$ can be surrounded by a sequence of discs of radius $r_{1}, r_{2}, \ldots, r_{n}$. Of course, if $r_{0}: r_{1} r_{2} r_{3} \cdots r_{n}$ is possible then so is $r_{0}: r_{n} r_{n-1} r_{n-2} \cdots r_{1}$ as well as any cyclic permutation of $r_{1} r_{2} r_{3} \cdots r_{n}$. We only list one possibility from each equivalence class. We refer to (9) as a "sequence for small discs" when $r_{0}=r$ and as a "sequence for large discs" when $r_{0}=1$. When there is only one possible sequence up to the symmetries just described, we say the sequence is unique.

We have already determined the solutions of (3) and thus the solutions of (8) with $r_{0}=r$. For each of the nine values of $r$ there are two possible sequences, $r: r r r r r$ and the sequence given in the last column of the table. We argue that $r: \operatorname{rrrrr}$ does not occur except when $r=c_{5}$. Suppose the configuration contains at least one small disc with sequence rrrrrr. Consider the small discs that are tangent to at least one small disc with sequence $r r r r r$. They cannot all have sequence $r r r r r$. So there is at least one small disc $D$ whose sequence is not $r r r r r$ but which is tangent to a small disc with sequence $r r r r r$. This implies that the sequence for $D$ must contain $r r r$ but not be equal to $r r r r r$. So it must contain $1 \mathrm{rrr}$ or $r r r 1$. From the table we see that this is possible only for $r=c_{5}$.

Finding the possible solutions of (8) with $r_{0}=1$ is straightforward. For each of the nine values of $r$ we compute $\alpha^{\prime}$ and $\beta$ and check for what choices of $r_{1}, r_{2}, \ldots, r_{n}$ (8) is satisfied with $r_{0}=1$. We list the possibilities as we consider each of the nine values of $r$.

For some values of $r$ we can give very explicit descriptions of all possible compact packings, but for others we can only show that there is a correspondence between packings and tilings using certain polygons. The order in which we consider the nine values of $r$ is for the sake of exposition. We start with $r=c_{6}$, the only value of $r$ for which the packing is unique. Of course, we can translate, rotate and reflect any compact packing to get another compact packing. When we say unique we implicitly mean unique up to these symmetries. Throughout the arguments that follow we freely use these symmetries to assume, for example, that a certain feature of the configuration is oriented in a particular direction.

For the next three values of $r$, the compact configurations are obtained by starting with a triangular lattice of large discs and either replacing some of the discs by groups of small discs $\left(r=c_{5}\right)$ or filling in some of the holes with small discs $\left(r=c_{8}, c_{9}\right)$. For the next three values, $c_{4}, c_{7}, c_{2}$, we can only show that there is a one to one correspondence between compact packings and tilings of the plane with polygons whose sides all have length 2 . For the last two values, $c_{1}, c_{3}$, we show that the packing is made of layers, but for each layer there is a twofold choice.

Possible Packings for $r=c_{6}$

There is a unique sequence for small discs and a priori two possible sequences for large discs:

$$
\begin{gathered}
r: 1 r r 1 r . \\
1: \text { rrrrrrrrrrrr } \text { or } 111111 .
\end{gathered}
$$


We argue that the $1: 111111$ sequence does not appear. The argument is similar to the argument used to rule out $r:$ rrrrrr. If $1: 111111$ appears, then there must be a large disc $D$ whose sequence is not 111111 but which is tangent to a large disc with sequence 111111. So the sequence of $D$ must contain 111 but not equal 111111 . This contradicts the above possible sequences around a large disc. Thus the only possible sequence around a large disc is rrrrrrrrrr.

We show that these unique sequences imply the configuration is unique. We start with a large disc which we label $a$ in Fig. 1(f). By the sequence for large discs it is surrounded by 12 small discs. Consider the small disc labeled $b$ in the figure. Up to symmetry, there is only one sequence that can surround it. Reflecting the configuration if necessary, we can assume that the discs around disc $b$ are as shown in the figure, i.e., discs $a, c, d, e, f$. Applying the sequence for small discs to disc $f$, we conclude that discs $g$ and $h$ must be present. Applying the sequence for small discs to disc $g$, we conclude that discs $i$ and $j$ are present. Note that we now have three large discs, $a, e$ and $i$, forming an equilateral triangle, and by the sequence for large discs each of them is surrounded by 12 small discs. We can now repeat this argument to fill out the configuration.

\section{Possible Packings for $r=c_{5}$}

This is the case for which there are two possible sequences for small discs. For the possible sequences for a large disc we give a number after the sequence to indicate the number of discs around the large disc. (This is just to aid in reading the following.)

$$
\begin{aligned}
& r: 1 r r r 1 \text { or rrrrr. } \\
& 1: r r 11111(7), \quad \operatorname{rrr} 1 r 111(8), \quad r r r 11 r 11(8), \quad r r 1 r r 111(8),
\end{aligned}
$$

rr11rr11(8), $\quad r r r 1 r 1 r 1(9), \quad \operatorname{rr} 1 r r 1 r 1(9), \quad r r 1 r r 1 r r 1(9)$ or 111111(6).

In the packing shown in Fig. 1(e) we can replace any hexagonal block of seven small discs by a single large disc. If we replace all such blocks of seven small discs, then the result will just be the triangular packing of large discs. If we start with the triangular packing by large discs, take a subset of the discs such that no two of them are tangent, and replace the discs in this subset by hexagonal blocks of seven small discs, then the resulting packing will be compact. We claim that all compact packings can be obtained in this fashion.

We begin by showing that every connected component of small discs must consist of seven small discs arranged in a hexagon like those in Fig. 1(e). A connected component of small discs must contain a small disc that is tangent to a large disc. We label this disc $a$ in Fig. 1(e). Since it is tangent to a large disc, there is up to symmetry only one possible sequence around it. We can assume the sequence is that shown in the figure, i.e., discs $b, c, d, e, f$. The possible sequences around $c, d$ and $e$ then imply that discs $g, h, i, j, m$ must be present. The possible sequences around $h$ then imply discs $k, l$ must be present. Thus small discs can only appear as part of a cluster of seven small discs arranged in a hexagon and surrounded by six large discs.

Now we replace every hexagonal block of seven small discs in our compact configuration by a single large disc. The result is a compact configuration with only large discs. The only such configuration is the triangular packing. Thus our original packing 
is obtained by replacing some subset of non-tangent large discs in the triangular packing by hexagonal blocks of seven small discs.

Possible packings for $r=c_{8}$

There is a unique sequence for small discs,

$$
r: 111
$$

and 13 sequences for large discs which we do not list. They are not needed in the argument.

The unique sequence for small discs says that each small disc is surrounded by three large discs that are tangent to each other. So if we remove the small discs, the configuration will still be compact. Thus we can remove all the small discs from our configuration and obtain a compact configuration with only large discs. There is only one such configuration, the triangular one. Thus we have shown that every compact configuration is given by putting small discs into some subset of the holes in the triangular lattice configuration of large discs. (Equivalently, every compact configuration can be obtained by removing some subset of the small discs from the configuration in Fig. 1(h).)

\section{Possible packings for $r=c_{9}$}

The sequence for small discs is unique:

$$
r: 11 r r
$$

We do not list the 195 possible sequences for large discs. Note that in the configuration in Fig. 1(i) we have a triangular lattice of large discs with each hole filled with a cluster of three small discs which are tangent to each other. We can remove any of the clusters of three tangent small discs and still have a compact configuration. We show that any compact configuration can be obtained in this way.

Start with a small disc. The sequence for small discs implies it is tangent to two other small discs which are tangent to each other. The sequence for small discs applied to these three small discs then implies that they sit inside the hole formed by three large discs that are tangent to each other. We can remove the three small discs and still have a compact configuration. Thus we can remove all the small discs and have a compact configuration with only large discs. It must be the triangular lattice configuration. So any compact configuration is formed by putting clusters of three small discs into some of the holes in the triangular lattice configuration of large discs. (Equivalently, we can form a compact configuration by removing some subset of the clusters of three small discs from Fig. 1(i).)

\section{Possible packings for $r=c_{4}$}

There is a unique sequence for small discs and four possible sequences for large discs:

$$
\begin{gathered}
r: 1111 . \\
1: r 1 r 1111, \quad r 11 r 111, \quad r 1 r 1 r 1 r 1 \quad \text { or } 111111 .
\end{gathered}
$$




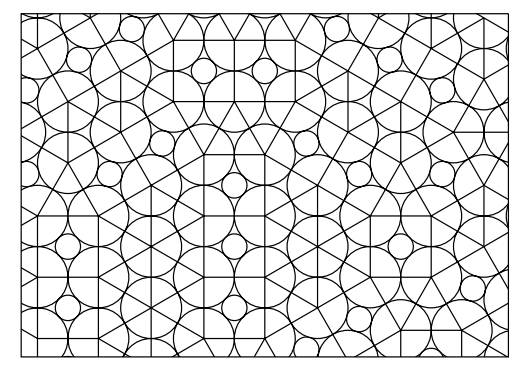

(a)

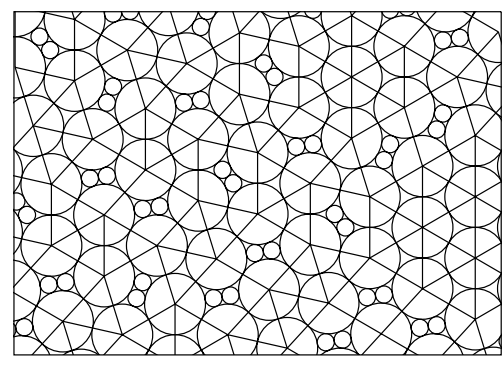

(b)

Fig. 2. Two compact packings and the corresponding tilings. (a) Tiling by squares and triangles for $r=c_{4}$. (b) Tiling by rhombi and triangles for $r=c_{7}$.

The sequence for small discs implies that every small disc is surrounded by four large discs whose vertices form a square with sides of length 2 . In the configuration in Fig. 1(d) these squares tile the plane. However, there are many compact packings in which they do not tile the plane. We show there is a one to one correspondence between compact packings with $r=c_{4}$ and tilings of the plane using squares with sides of length 2 and equilateral triangles with sides of length 2 . An example of a packing and the corresponding tiling is shown in Fig. 2(a).

Given a packing, we draw line segments between the centers of any two large discs that are tangent. These line segments, which all have length 2 , divide the plane into squares and equilateral triangles. To see this, consider a large disc and the possible sequences around it. For the sequence $r 1 r 1111$, the large disc is a vertex of two adjacent squares and three triangles. For the sequence $r 11 r 111$, the large disc is again a vertex of two squares and three triangles, but now the two squares do not share a side. For the sequence $r 1 r 1 r 1 r 1$, the large disc is a vertex of four squares. For the sequence 111111 the large disc is a vertex of six triangles. So in each case the center of the large disc is a vertex of squares and triangles that fit together to tile the space around the large disc.

Thus the packing can be used to construct a tiling by squares and triangles. Conversely, given such a tiling, we can construct a compact packing by putting a large disc at the vertices of the squares and triangles and then putting a small disc at the center of each square.

\section{Possible Packings for $r=c_{7}$}

The sequence for small discs is unique and there are six sequences for larges discs:

$$
\begin{aligned}
& r: 111 r . \\
& 1: r r 1 r 1111(8), \quad r r 11 r 111(8), \quad r r r 1 r 1 r 1 r 1(10), \\
& r r 1 r r 1 r 1 r 1(10), \quad r r 1 r 1 r r 1 r 1(10) \quad \text { or } 111111(6) .
\end{aligned}
$$

The third of the possibilities for a large disc implies that there is a small disc that is tangent to two other small discs. This contradicts the sequence for small discs. So the third possibility for large discs does not occur. 
The sequence for small discs implies that each small disc is tangent to one other small disc and this pair is surrounded by four large discs. The centers of these four large discs are the vertices of a rhombus whose sides are of length 2 and whose acute angle is $2 \cos ^{-1}((-1+\sqrt{17}) / 4)$. Small discs can only appear as pairs inside such rhombi. In the packing shown in Fig. 1(g) these rhombi tile the plane.

There are many possible compact packings for $r=c_{7}$. We claim there is a one to one correspondence between compact packings and tilings of the plane using these rhombi and equilateral triangles with sides of length 2 . An example of a packing and the corresponding tiling is shown in Fig. 2(b). Given a packing we construct the tiling by drawing line segments between the centers of any pair of large discs that are tangent to each other. To see that this produces a tiling of the plane by rhombi and triangles, we can argue as we did in the case of $r=c_{4}$. We consider the possible sequences for a large disc and see that in each case the center of the disc is a vertex of rhombi and triangles that fit together to tile the space around the disc. Given a tiling we construct a packing by putting a large disc at each vertex of each triangle and each rhombi. Then we put a pair of small discs inside each rhombi.

Possible Packings for $r=c_{2}$

The sequence for small discs is unique, and there are three possible sequences for large discs:

$$
\begin{gathered}
r: 111 r r . \\
1: r r 1 r 111, \quad r 11 r 11 r \quad \text { or } 111111 .
\end{gathered}
$$

In addition to the packing in Fig. 1(b), we give a second example in Fig. 3(a). As in the previous two cases we show that the possible compact packings are equivalent to a tiling problem. In both of the figures the small discs all appear in clusters of three. We argue that this property is true in any compact packing. The sequence for small discs implies that every small disc is tangent to two other small discs which are tangent to each other. The sequence for small discs applied to these three small discs then implies that they are surrounded by six large discs. The vertices of these six large discs form a six-sided polygon with sides of length 2. We have drawn one in Fig. 1(b).

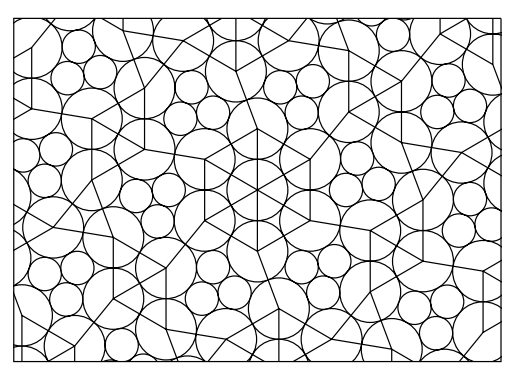

(a) $r=c_{2}$

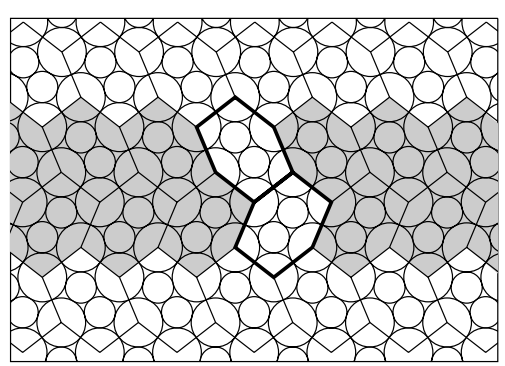

(b) $r=c_{1}$

Fig. 3. Two more compact packings. 
We construct the tilings in the same way as in the previous two cases. We draw a line segment between the centers of any two discs of radius 2 that are tangent. These lines form equilateral triangles in addition to the six-sided polygons. The same arguments used in the previous cases show that given a packing, the six-sided polygons and triangles tile the plane and, conversely, given such a tiling we can construct a compact packing.

Possible Packings for $r=c_{1}$

The possible sequences are

$$
\begin{gathered}
r: 1111 r \\
1: r 1 r 1 r 1 r \quad \text { or } 111111 .
\end{gathered}
$$

Start with a small disc. The unique sequence for small discs implies it touches one other small disc. By the sequence for small discs, this pair of tangent small discs is surrounded by six large discs. The centers of these six large discs are the vertices of a flattened hexagon. (An example of the flattened hexagon is shown in Fig. 1(a).) We show that these flattened hexagons tile the plane.

The flattened hexagon has two different angles in it which we call $\theta_{1}$ and $\theta_{2}$ with $\theta_{1}<\theta_{2}$. We have four $\theta_{1}$ 's and two $\theta_{2}$ 's in the flattened hexagon. The sequence for large discs implies that at every large disc we must have two $\theta_{1}$ 's and one $\theta_{2}$. It follows that the flattened hexagons tile the plane.

To determine the possible tilings, we consider two cases. The first case is that all the flattened hexagons have the same orientation. The second case is that they do not. It is easy to see that in the first case the configuration must be the one shown in Fig. 1(a). In the second case there must be two flattened hexagons that share a side but have different orientations. So we have two flattened hexagons which fit together as shown by the two bold hexagons in Fig. 3(b). Using the constraint that each large disc has two $\theta_{1}$ 's and one $\theta_{2}$, it follows that all the shaded hexagons must be present, extending to infinity in both directions.

Next we consider what can happen above and below this shaded layer. Consider adding a flattened hexagon with two sides adjacent to the shaded layer. There are two possible orientations, but as soon as we add one flattened hexagon, the orientation of all the other ones touching this boundary of the shaded region must be the same. In the figure we have added one above the shaded region with the same orientation as the shaded flattened hexagons along this upper boundary. Below the shaded region we have added one whose orientation is different from the shaded flattened hexagons along the lower boundary. It follows that the configuration must consist of such layers with the freedom to chose an orientation for each layer.

Possible Packings for $r=c_{3}$

The sequence for small discs is unique, and there are five possible sequences for large discs:

$$
\begin{aligned}
r: 1 r 1 r 1 . & \\
1: \operatorname{rrr} 1111, \quad \operatorname{rrrr} 1 r 1, \quad & \operatorname{rrrr} 1 r r 1, \quad \operatorname{rr} 1 r r r 1 \quad \text { or } 111111 .
\end{aligned}
$$




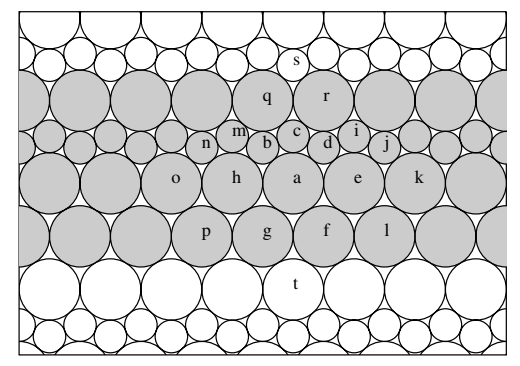

(a)

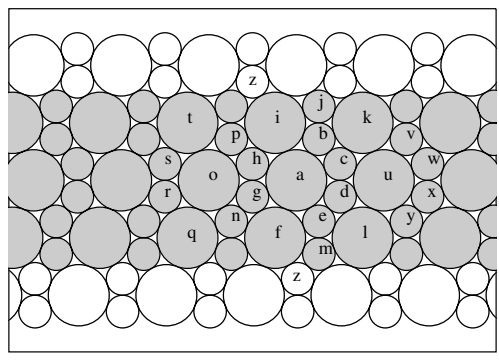

(b)

Fig. 4. Examples of the two types of compact packings for $r=c_{3}$. (a) Example of the first class. (b) Example of the second class.

We show that there are two types of compact configurations for this value of $r$. Figure 4(a) gives an example from the first class. In general, a configuration in this class is formed of layers of large discs and layers of small discs (which oscillate up and down slightly as in the figure). The only constraint on the sequence of layers is that we cannot have two adjacent layers of small discs. An example from the second class is shown in Fig. 4(b). These configurations are formed by layers made up of large discs alternating with two tangent small discs aligned vertically. (For example, the shaded discs in Fig. 4(b) consist of three layers.) Each layer has two possible locations relative to the layer above (or below) it. Note that the configuration in Fig. 1(c) belongs to both classes and is the only configuration that does.

We start by showing that the sequence $1: \operatorname{rrrr} 1 r 1$ does not occur. Consider the small disc represented by the last $r$. It touches the large disc at the center of the sequence, and there are two other large discs tangent to the small disc and the large disc at the center. So the sequence around the small disc must contain 111 which is not possible. So the sequence 1 : $\operatorname{rrrr} 1 r 1$ does not occur.

We now divide into two cases. The first case is that at least one large disc has the sequence $1: \operatorname{rr} 1111$. The second case is that no large disc has this sequence. We show that in the first case the configuration is of the first type described above, and in the second case it is of the second type.

In Fig. 4(a) the large disc that is assumed to have the sequence $1: \operatorname{rr} 1111$ is labeled $a$. It is surrounded by $b, c, d, e, f, g, h$. Using the possible sequences, we now have

$$
\begin{aligned}
& e \Rightarrow i, j, k, l, \\
& h \Rightarrow m, n, o, p, \\
& c \Rightarrow q, r .
\end{aligned}
$$

The large discs $e$ and $h$ are now surrounded by the same sequence that $a$ is. Repeating this argument, we conclude that all of the shaded discs are present. We now consider what can happen above and below the shaded discs. Consider two large shaded discs that are tangent to each other and at the edge of the shaded discs. They must both be tangent to a disc that is not shaded. It is either small or large. The case of a small disc is shown 
by $s$ above the shaded discs, and the case of a large disc is shown by $t$ below the shaded discs. It is now straightforward to show that $s$ forces the layer of small unshaded discs just above the shaded discs as well as the layer of large unshaded discs at the top edge of the figure. The small disc $t$ is easily shown to imply the layer of large unshaded discs just below the shaded discs.

For the second case we refer to Fig. 4(b). In the second case we are assuming that the sequence $1: \operatorname{rr} 1111$ does not occur. It is easy to see that this implies that if the configuration contains at least one small disc, then the sequence $1: 111111$ cannot occur. So the only possible sequences around a large disc are $\operatorname{rrr} 1 \mathrm{rr} 1$ and $\operatorname{rrr} 1 \mathrm{rrr} 1$. If the sequence $\operatorname{rrr} 1 \operatorname{rr} 1$ never appears, then it is easy to show that the configuration must be that shown in Fig. 1(c). So we now assume there is at least one large disc with the sequence $\operatorname{rrr} 1 \operatorname{rr} 1$. In Fig. 4(b) this disc is labeled $a$, and the discs surrounding it $b, c, d, e, f, g, h, i$. We now have

$$
\begin{aligned}
b & \Rightarrow j, k, \\
e & \Rightarrow l, m, \\
g & \Rightarrow n, o, \\
h & \Rightarrow p, \\
o & \Rightarrow q, r, s, t, \\
c & \Rightarrow u, \\
u & \Rightarrow v, w, x, y .
\end{aligned}
$$

This shows that the large discs $o$ and $u$ have the same sequence around them as the large disc $a$. So we can repeat this argument and conclude that all of the shaded discs must be present.

We now ask what can happen above and below the shaded discs in Fig. 4(b). The large discs $i$ must touch one more large disc and one more small disc in addition to the shaded discs. There are two possible locations for the additional small disc.

One possibility is given by the disc $z$ just above the shaded region, and the other possibility by the disc $z$ just below the shaded region. It is now easy to show that the presence of the $z$ disc just above the shaded discs forces all of the layer of unshaded discs above the shaded ones. The presence of $z$ as shown below the shaded discs forces the layer of unshaded discs below the shaded ones. We conclude that the configuration in the second case is made up of layers. (In the figure there are three layers with shaded discs and single layers above and below them that are unshaded.) Each layer has two possible locations relative to the layer below (or above) it.

\section{References}

1. G. Blind and R. Blind, Packings of unequal circles in a convex set, Discrete Comput. Geom. 28 (2002), $115-119$.

2. L. Fejes Tóth, Regular Figures, Pergamon Press, Oxford, 1964.

3. L. Fejes Tóth, Compact packing of circles, Studia Sci. Math. Hungar. 19 (1984), 103-107.

4. A. Florian, Geometry of circular layering, Acta Math. Acad. Sci. Hungar. 18 (1967), 341-358.

5. A. Heppes, On the densest packing of discs of radius 1 and $\sqrt{2}-1$, Studia Sci. Math. Hungar. 36 (2000), $433-454$. 
6. A. Heppes, Some densest two-size disc packings in the plane, Discrete Comput. Geom. 30 (2003), 241-262.

7. A. Heppes and J. Molnár, Újabb eredmények a diszkrét geometriában, Mat. Lapok 11 (1960), 330-355.

8. C. N. Likos and C. L. Henley, Complex alloy phases for binary hard-disc mixtures, Philos. Mag. B 68 (1993), 85-113.

9. J. Molnár, Unterdeckung und Überdeckung der Ebene durch Kreise, Ann. Univ. Sci. Budapest 2 (1959), $33-40$.

Received August 30, 2004, and in revised form January 10, 2005. Online publication May 20, 2005. 